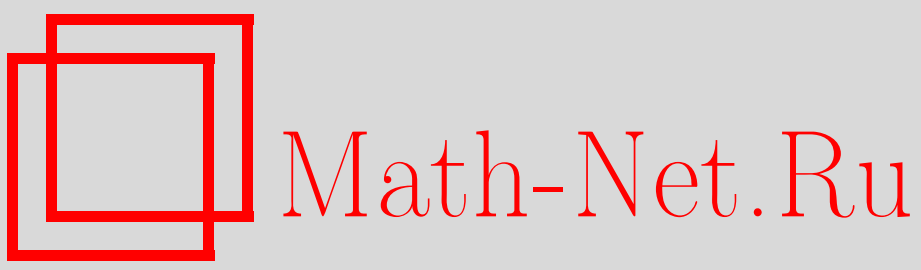

В. А. Краснов, О группе Брауэра вещественной алгебраической поверхности, Матем. заметки, 1996, том 60, выпуск 6, 935-938

DOI: https://doi.org/10.4213/mzm1913

Использование Общероссийского математического портала MathNet.Ru подразумевает, что вы прочитали и согласны с пользовательским соглашением

http://www. mathnet.ru/rus/agreement

Параметры загрузки:

IP : 3.89 .197 .203

26 апреля 2023 г., 15:41:18

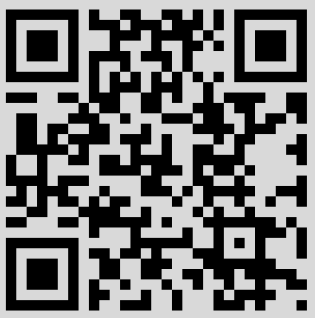




\section{О ГРУППЕ БРАУЭРА ВЕЩЕСТВЕННОЙ АЛГЕБРАИЧЕСКОЙ ПОВЕРХНОСТИ}

\section{В. А. Краснов}

Введение. Далее $X$ - неособая полная вещественная алгебраическая поверхность, тогда группа Брауэра $\operatorname{Br}(X)$ равна когомологической группе Брауэра $\operatorname{Br}^{\prime}(X)=H_{\mathrm{et}}^{2}\left(X, \mathbb{G}_{m}\right)$ (см. [1]). В работе [2] было доказано равенство

$$
\operatorname{Br}^{\prime}(X)=(\mathbb{Q} / \mathbb{Z})^{\rho_{0}(X)} \oplus H^{3}(X(\mathbb{C}) ; G, \mathbb{Z}-)_{\text {tors }} .
$$

В этом равенстве $\rho_{0}(X)$ - число Лефшеца, которое равно размерности коядра гомоморфизма

$$
(\operatorname{Pic} X) \otimes \mathbb{Q} \rightarrow H^{2}(X(\mathbb{C}), \mathbb{Q})_{-}
$$

причем $H^{2}(X(\mathbb{C}), \mathbb{Q})_{\text {_ }}$ - подпространство $H^{2}(X(\mathbb{C}), \mathbb{Q})$, антиинвариантное относительно инволюции комплексного сопряжения $g: X(\mathbb{C}) \rightarrow X(\mathbb{C}) ; G-$ группа второго порядка, порожденная инволюцией $g ; \mathbb{Z}_{-}$- постоянный пучок на $X(\mathbb{C})$ со слоем $\mathbb{Z}$, инволюция $g$ на котором действует умножением на $-1 ; H^{3}(X(\mathbb{C}) ; G, \mathbb{Z}-)_{\text {tors }}-$ подгруппа кручения эквивариантной группы когомологий. Из (1), в частности, получаем следующее равенство:

$$
\operatorname{dim}_{2} \operatorname{Br}(X)=\rho_{0}(X)+\operatorname{dim}_{2} H^{3}(X(\mathbb{C}) ; G, \mathbb{Z}-),
$$

где ${ }_{2} \operatorname{Br}(X),{ }_{2} H^{3}(X(\mathbb{C}) ; G, \mathbb{Z}-)$ - 2-элементарные подгруппы. В работах [2]-[4] имеются частные результаты о размерности $\operatorname{dim}_{2} \operatorname{Br}(X)$, но окончательного ответа еще не было. Здесь мы доказываем следующий результат.

Теорема 1. Пусть $X(\mathbb{R}) \neq \varnothing$, тогда выполняется равенство

$$
\operatorname{dim}_{2} \operatorname{Br}(X)=\rho_{0}(X)-q(X)+k^{1}(Y)+2 s-1,
$$

әде $q(X)$ - иррегулярность $X, Y=X(\mathbb{C}) / G$ и $k^{1}(Y)$ равно размерности ядра гомоморфизма ограничения $H^{1}\left(Y, \mathbb{F}_{2}\right) \rightarrow H^{1}\left(X(\mathbb{R}), \mathbb{F}_{2}\right), s$ - число компонент связности мнохсества $X(\mathbb{R})$.

Вычисление всей группы Брауэра $\operatorname{Br}(X)$ является задачей более сложной. Заметим сначала, что если $H_{1}(X(\mathbb{C}), \mathbb{Z})=0$, то в [2] доказано равенство

$$
\operatorname{Br}(X)=(\mathbb{Q} / \mathbb{Z})^{\rho_{0}(X)} \oplus(\mathbb{Z} / 2)^{2 s-1} .
$$

В этой заметке мы вычисляем группу Брауэра поверхности в случае, когда $H_{1}(X(\mathbb{C}), \mathbb{Z})=\mathbb{Z} / 2$. Соответствующая теорема обобщает резульат о группе Брауэра поверхности Энриквеса в [2]. Прежде чем сформулировать эту теорему, сделаем несколько замечаний.

Так как $H_{1}(X(\mathbb{C}), \mathbb{Z})=\mathbb{Z} / 2$, то $H^{1}\left(X(\mathbb{C}), \mathbb{F}_{2}\right)=\mathbb{F}_{2}$, образующую группы $H^{1}\left(X(\mathbb{C}), \mathbb{F}_{2}\right)$ обозначим через $\theta$. Гомоморфизм $H^{1}\left(Y, \mathbb{F}_{2}\right) \rightarrow H^{1}\left(X(\mathbb{C}), \mathbb{F}_{2}\right)$ является вложением при $X(\mathbb{R}) \neq \varnothing$, поэтому тогда группа $H^{1}\left(Y, \mathbb{F}_{2}\right)$ может быть равна либо 0 , либо $\mathbb{F}_{2}$. 
ТЕОРемА 2. Пусть $H_{1}(X(\mathbb{C}), \mathbb{Z})=\mathbb{Z} / 2 u X(\mathbb{R}) \neq \varnothing$, тогда справедливь утверждения:

1) $\left.е с л и \theta\right|_{X(\mathbb{R})} \neq 0$, mo $\operatorname{Br}(X)=(\mathbb{Q} / \mathbb{Z})^{\rho_{0}(X)} \oplus(\mathbb{Z} / 2)^{2 s-1}$

2) $\left.е c л u \theta\right|_{X(\mathbb{R})}=0, H^{1}\left(Y, \mathbb{F}_{2}\right) \neq 0, m o \operatorname{Br}(X)=(\mathbb{Q} / \mathbb{Z})^{\rho_{0}(X)} \oplus(\mathbb{Z} / 2)^{2 s}$;

3) если $\left.\theta\right|_{X(\mathbb{R})}=0, H^{1}\left(Y, \mathbb{F}_{2}\right)=0, \operatorname{mo} \operatorname{Br}(X)=(\mathbb{Q} / \mathbb{Z})^{\rho_{0}(X)} \oplus(\mathbb{Z} / 2)^{2 s-2} \oplus \mathbb{Z} / 4$.

ДоКАЗАТЕЛЬСТВо ТЕОРЕМ. В силу равенства (2) для доказательства теоремы 1 достаточно установить равенство

$$
\operatorname{dim}_{2} H^{3}\left(X(\mathbb{C}) ; G, \mathbb{Z}_{-}\right)=k^{1}(Y)-q(X)+2 s-1 .
$$

Покажем сначала, что

$\operatorname{dim}_{2} H^{3}\left(X(\mathbb{C}) ; G, \mathbb{Z}_{-}\right)=\operatorname{dim} H^{3}\left(X(\mathbb{C}) ; G, \mathbb{F}_{2}\right)-\operatorname{dim} H^{1}\left(X(\mathbb{R}), \mathbb{F}_{2}\right)-q(X)$ Для этого заметим, что

$$
\begin{aligned}
\operatorname{dim}_{2} H^{3}\left(X(\mathbb{C}) ; G, \mathbb{Z}_{-}\right)= & \operatorname{dim} H^{3}\left(X(\mathbb{C}) ; G, \mathbb{Z}_{-}\right) \otimes \mathbb{F}_{2} \\
& -\operatorname{rk} H^{3}\left(X(\mathbb{C}) ; G, \mathbb{Z}_{-}\right),
\end{aligned}
$$

$\operatorname{dim} H^{3}\left(X(\mathbb{C}) ; G, \mathbb{Z}_{-}\right) \otimes \mathbb{F}_{2}=\operatorname{dim} H^{3}\left(X(\mathbb{C}) ; G, \mathbb{F}_{2}\right)-\operatorname{dim}_{2} H^{4}\left(X(\mathbb{C}) ; G, \mathbb{Z}_{-}\right)$,

$\operatorname{rk} H^{3}\left(X(\mathbb{C}) ; G, \mathbb{Z}_{-}\right)=\operatorname{dim} H^{3}\left(X(\mathbb{C}) ; G, \mathbb{Z}_{-}\right) \otimes \mathbb{Q}$

$$
=\operatorname{dim} H^{3}\left(X(\mathbb{C}) ; G, \mathbb{Q}_{-}\right)=\operatorname{dim} H^{3}(X(\mathbb{C}), \mathbb{Q})_{-} .
$$

Осталось отметить, что гомоморфизм ограничения

$$
\beta: H^{4}\left(X(\mathbb{C}) ; G, \mathbb{Z}_{-}\right) \rightarrow H^{4}\left(X(\mathbb{R}) ; G, \mathbb{Z}_{-}\right)=H^{1}\left(X(\mathbb{R}), \mathbb{F}_{2}\right)
$$

является изоморфизмом (см. [5]), поэтому

$$
\operatorname{dim}_{2} H^{4}\left(X(\mathbb{C}) ; G, \mathbb{Z}_{-}\right)=\operatorname{dim} H^{1}\left(X(\mathbb{R}), \mathbb{F}_{2}\right),
$$

а также имеем равенство

$$
\operatorname{dim} H^{3}(X(\mathbb{C}), \mathbb{Q})_{-}=q(X) .
$$

Итак, соотношение (4) доказано, следовательно, чтобы установить формулу (3), достаточно показать, что

$$
\operatorname{dim} H^{3}\left(X(\mathbb{C}) ; G, \mathbb{F}_{2}\right)-\operatorname{dim} H^{1}\left(X(\mathbb{R}), \mathbb{F}_{2}\right)=k^{1}(Y)+2 s-1
$$

Для доказательства этого равенства заметим, что гомоморфизм ограничения

$$
\beta: H^{3}\left(X(\mathbb{C}) ; G, \mathbb{F}_{2}\right) \rightarrow H^{3}\left(X(\mathbb{R}) ; G, \mathbb{F}_{2}\right)=H^{*}\left(X(\mathbb{R}), \mathbb{F}_{2}\right)
$$

дополняется до следующей точной последовательности (см. [5])

$$
H^{1}\left(X(\mathbb{R}), \mathbb{F}_{2}\right) \stackrel{j^{*}}{\rightarrow} H^{3}\left(Y, \mathbb{F}_{2}\right) \stackrel{i^{*}}{\rightarrow} H^{3}\left(X(\mathbb{C}) ; G, \mathbb{F}_{2}\right) \stackrel{\beta}{\rightarrow} H^{*}\left(X(\mathbb{R}), \mathbb{F}_{2}\right) \rightarrow \mathbb{F}_{2} \rightarrow 0
$$

где $j^{*}$ - гомоморфизм Гизина, из которой вытекает равенство (5). Итак, теорема 1 доказана, перейдем к доказательству теоремы 2 .

Согласно равенству (1) достаточно установить утверждения:

$\left.1^{\prime}\right)$ если $\left.\theta\right|_{X(\mathbb{R})} \neq 0$, то $H^{3}(X(\mathbb{C}) ; G, \mathbb{Z}-)_{\text {tors }}=(\mathbb{Z} / 2)^{2 s-1}$;

$\left.2^{\prime}\right)$ если $\left.\theta\right|_{X(\mathbb{R})}=0, H^{1}\left(Y, \mathbb{F}_{2}\right) \neq 0$, то $H^{3}(X(\mathbb{C}) ; G, \mathbb{Z}-)_{\text {tors }}=(\mathbb{Z} / 2)^{2 s} ;$

$\left.3^{\prime}\right)$ если $\left.\theta\right|_{X(\mathbb{R})}=0, H^{1}\left(Y, \mathbb{F}_{2}\right)=0$, то $H^{3}\left(X(\mathbb{C}) ; G, \mathbb{Z}_{-}\right)_{\text {tors }}=(\mathbb{Z} / 2)^{2 s-2} \oplus \mathbb{Z} / 4$. 
Для вычисления группы $H^{3}\left(X(\mathbb{C}) ; G, \mathbb{Z}_{-}\right)_{\text {tors }}$ рассмотрим гомоморфизм ограничения

$$
\beta: H^{3}\left(X(\mathbb{C}) ; G, \mathbb{Z}_{-}\right) \rightarrow H^{3}\left(X(\mathbb{R}) ; G, \mathbb{Z}_{-}\right)=H^{\mathrm{ev}}\left(X(\mathbb{R}), \mathbb{F}_{2}\right)
$$

где $H^{\mathrm{ev}}\left(X(\mathbb{R}), \mathbb{F}_{2}\right)=H^{0}\left(X(\mathbb{R}), \mathbb{F}_{2}\right) \oplus H^{2}\left(X(\mathbb{R}), \mathbb{F}_{2}\right)$. Этот гомоморфизм дополняется до следующей точной последовательности (см. [5])

$$
H^{1}\left(X(\mathbb{R}), \mathbb{F}_{2}\right) \stackrel{j^{\prime *}}{\rightarrow} H^{3}\left(Y, \mathbb{Z}^{\prime}\right) \stackrel{i^{*}}{\rightarrow} H^{3}\left(X(\mathbb{C}) ; G, \mathbb{Z}_{-}\right) \stackrel{\beta}{\rightarrow} H^{\mathrm{ev}}\left(X(\mathbb{R}), \mathbb{F}_{2}\right) \rightarrow \mathbb{F}_{2}
$$

где $\mathbb{Z}^{\prime}=f_{*}^{G}\left(\mathbb{Z}_{-}\right), f: X(\mathbb{C}) \rightarrow Y$ - проекция, а $j^{\prime *}$ - аналог гомоморфизма Гизина, он устроен следующим образом. Пусть $a=[\Gamma]^{*} \in H^{1}\left(X(\mathbb{R}), \mathbb{F}_{2}\right)$, где $\Gamma$ кривая на $X(\mathbb{R}), U$ - замкнутая $G$-инвариантная окрестность $\Gamma$ в $X(\mathbb{C}), V=U / G$, тогда группа $H^{3}\left(V, \partial V ; \mathbb{Z}^{\prime}\right)$ равна $\mathbb{F}_{2}$. Обозначим образ образующей этой группы в $H^{3}\left(Y, \mathbb{Z}^{\prime}\right)$ через $A$, тогда $j^{\prime *}(a)=A$. Вычислим теперь группу $H^{3}\left(Y, \mathbb{Z}^{\prime}\right)$. Для этого рассмотрим точную последовательность пучков на $Y$

$$
0 \longrightarrow \mathbb{Z} \longrightarrow f_{*}\left(\mathbb{Z}_{-}\right) \stackrel{1+g^{*}}{\longrightarrow} \mathbb{Z}^{\prime} \longrightarrow 0
$$

Так как гомоморфизм $H^{q}(Y, \mathbb{Z}) \rightarrow H^{q}\left(Y, f_{*}(\mathbb{Z})\right)$ совпадает с гомоморфизмом $f^{*}: H^{q}(Y, \mathbb{Z}) \rightarrow H^{q}(X(\mathbb{C}), \mathbb{Z})$, то из этой точной последовательности и из наших предположений следует, что гомоморфизм

$$
1+g^{*}: H^{3}\left(Y, f_{*}\left(\mathbb{Z}_{-}\right)\right) \rightarrow H^{3}\left(Y, \mathbb{Z}^{\prime}\right)
$$

является изоморфизмом (см. [2]). Таким образом, имеем равенства

$$
H^{3}\left(Y, \mathbb{Z}^{\prime}\right)=H^{3}\left(Y, f_{*}\left(\mathbb{Z}_{-}\right)\right)=H^{3}(X(\mathbb{C}), \mathbb{Z})=H^{3}\left(X(\mathbb{C}), \mathbb{F}_{2}\right)=\mathbb{F}_{2}
$$

Если отождествить согласно этим равенствам группу $H^{3}\left(Y, \mathbb{Z}^{\prime}\right)$ с $H^{3}\left(X(\mathbb{C}), \mathbb{F}_{2}\right)$, то из определения гомоморфизма $j^{\prime *}$ следует, что он совпадает с гомоморфизмом Гизина

$$
j^{*}: H^{1}\left(X(\mathbb{R}), \mathbb{F}_{2}\right) \rightarrow H^{3}\left(X(\mathbb{C}), \mathbb{F}_{2}\right)
$$

Теперь мы можем доказать утверждение $\left.1^{\prime}\right)$. Действительно, если $\left.\theta\right|_{X(\mathbb{R})} \neq 0$, то гомоморфизм (8) эпиморфен, поэтому эпиморфен гомоморфизм $j^{* *}$, а тогда из точной последовательности (7) следует равенство

$$
H^{3}(X(\mathbb{C}) ; G, \mathbb{Z}-)=(\mathbb{Z} / 2)^{2 s-1} .
$$

Предположим теперь, что $\left.\theta\right|_{X(\mathbb{R})}=0$, тогда рассмотрим гомоморфизм редукции по $\bmod 2$

$$
R: H^{3}\left(X(\mathbb{C}) ; G, \mathbb{Z}_{-}\right) \rightarrow H^{3}\left(X(\mathbb{C}) ; G, \mathbb{F}_{2}\right)
$$


он продолжается до гомоморфизма точной последовательности (6) в точную последовательность (7), т.е. имеем коммутативную диаграмму

$$
\begin{aligned}
H_{1}\left(X(\mathbb{R}), \mathbb{F}_{2}\right) \stackrel{j^{\prime *}}{\longrightarrow} H^{3}\left(Y, \mathbb{Z}^{\prime}\right) \stackrel{i^{*}}{\longrightarrow} H^{3}\left(X(\mathbb{C}) ; G, \mathbb{Z}_{-}\right) & \downarrow R \\
H_{1}\left(X(\mathbb{R}), \mathbb{F}_{2}\right) \stackrel{j^{*}}{\longrightarrow} H^{3}\left(Y, \mathbb{F}_{2}\right) \stackrel{i^{*}}{\longrightarrow} & H^{3}\left(X(\mathbb{C}) ; G, \mathbb{F}_{2}\right) \\
\stackrel{\beta}{\longrightarrow} H^{\operatorname{ev}}\left(X(\mathbb{R}), \mathbb{F}_{2}\right) \rightarrow \mathbb{F}_{2} \rightarrow 0 & \| \\
& \stackrel{\beta}{\longrightarrow} H^{*}\left(X(\mathbb{R}), \mathbb{F}_{2}\right) \rightarrow \mathbb{F}_{2} \longrightarrow 0
\end{aligned}
$$

Рассмотрим гомоморфизм $H^{3}\left(Y, \mathbb{Z}^{\prime}\right) \rightarrow H^{3}\left(Y, \mathbb{F}_{2}\right)$ в ней. Если отождествить группу $H^{3}\left(Y, \mathbb{Z}^{\prime}\right)$ с $H^{3}\left(X(\mathbb{C}), \mathbb{F}_{2}\right)$, то этот гомоморфизм совпадает с гомоморфизмом $H^{3}\left(X(\mathbb{C}), \mathbb{F}_{2}\right) \rightarrow H^{3}\left(Y, \mathbb{F}_{2}\right)$, который называют трансфером. Он будет эпиморфизмом. Используя этот факт, теперь нетрудно вывести из диаграммы (9) утверждения $\left.2^{\prime}\right), 3^{\prime}$ ).

Ярославль

\section{СПИСОК ЦИТИРОВАННОЙ ЛИТЕРАТУРЫ}

1. Grothendieck A. // Le groupe de Brauer. Dix Exposes sur la Cohomologie des Schemas. Amsterdam: North-Holland, 1968. P. 46-188. 2. Краснов В. А. // Изв. РАН. Сер. матем. 1996. Т. 60. № 5. C. 57-88. 3. Nikulin V. V. // J. Reine Angew. Math. 1993. V. 444. P. 115-154. 4. Nikulin V. V. // Algebraic geometry and its applications. Proc. of the 8th Algebraic Geometry Conference. Yaroslavl', 1992. С. 113-136. 5. Краснов В. А. Эквивариантные когомологии вещественной алгебраической поверхности и их приложения // Изв. РАН. Сер. матем. 1997. Т. 61 (в печати). 